\title{
The role of the current profile in the improved H-mode scenario in ASDEX Upgrade
}

\author{
J Stober ${ }^{1}$, A.C.C. Sips ${ }^{1}$, C. Angioni ${ }^{1}$, C.B. Forest ${ }^{2}$, O. Gruber ${ }^{1}$, \\ J. Hobirk ${ }^{1}$, L.D. Horton ${ }^{1}$, C.F. Maggi ${ }^{1}$, M. Maraschek ${ }^{1}$, P. Martin ${ }^{3}$, \\ P.J. Mc Carthy ${ }^{4}$, V. Mertens ${ }^{1}$, Y.-S. Na ${ }^{5}$, M. $\operatorname{Reich}^{1}$, A. Stäbler ${ }^{1}$, \\ G. Tardini ${ }^{1}$, H. Zohm ${ }^{1}$, and the ASDEX Upgrade Team \\ ${ }^{1}$ MPI für Plasmaphysik, EURATOM Association, Garching, GERMANY \\ ${ }^{2}$ Dept. of Physics, University of Wisconsin, Madison, USA \\ ${ }^{3}$ Consorzio RFX, Associazione Euratom-ENEA sulla Fusione, Padova, Italy \\ ${ }^{4}$ Dept. of Physics, University College Cork, Assosiation EURATOM-DCU, Cork, Ireland \\ ${ }^{5}$ National Fusion Research Center, Yusung-Gu, Daejeon, Korea
}

\begin{abstract}
Recent studies at ASDEX Upgrade aim to further characterise and understand the physics of the improved H-mode scenario. The main focus is on the influence of the rampup phase of the plasma current and heating on energy confinement and MHD-activity during the subsequent flat-top phase. Depending on the ramp-up scenario two different stationary plasmas can be generated, which show different equilibrated current profiles, although external control parameters are the same in the flat-top phase. The difference of the current profiles in the flat-top phase seems to be due to different MHD modes. These MHD modes set in during relaxation of the current profile, which itself depends on the ramp-up scenario. Also the stored energy is different in the two cases as is the peaking of the temperature profiles.

Three mechanisms seem to play a role in linking the observed changes in MHD-behaviour and current profile to the changes of the kinetic profiles: the increased transport due to the MHD modes themselves, the variation of the ratio of magnetic shear $s$ to safety factor $q$, which modifies the critical temperature gradient-length for the onset of ITGs and effects of the H-mode pedestal pressure.
\end{abstract}




\section{Introduction}

The 'improved H-mode' scenario of ASDEX Upgrade [1] is a candidate for the ITER hybrid scenario and has been confirmed on several other devices [2, 3, 4]. Hybrid operation in ITER aims to increase the neutron fluence in prolonged discharges reducing the plasma current whilst maintaining a sufficient fraction of self-heating by $\alpha$-particles (although $Q<10$ ). Additionally, hybrid operation allows higher values of $q_{95}>3.0$, such that sawteeth and neoclassical-tearing-modes (NTMs) may be avoided and edge-localised-modes ELMs are

less severe, facilitating the routine operation of these plasma [5]. Recent results show that the improved H-mode scenario on ASDEX Upgrade and similar scenarios on other devices have beneficial properties with respect to energy confinement and MHD-stability, such that operation at the target fraction of self-heating $(Q=10)$ will be possible even with $q_{95} \approx 4.0$, when operated close to their limit of $\beta_{N} \approx 3.0$ [6]. These high $\beta$-plasma at $q_{95}>3.0$ are usually referred to as the hybrid-scenario. Alternatively the improved H-mode scenario can also be applied for $q_{95}=3.0$ such that, at full plasma current, the base line performance of ITER could be significantly exceeded $\left(Q>10, P_{f u s}>400 \mathrm{MW}\right)[7]$.

For this specific scenario, the results from different devices show many similarities. Still there are also significant differences and debate remains on which ingredients are essential for Hybrid operation ([2, 3, 4, 6, 7] and references therein). Hybrid scenarios typically apply moderate additional heating during the plasma current ramp-up to allow the formation of an adequate current profile (low central shear) for stronger heating in the flat-top phase. This main heating phase itself usually starts with a power level well above the threshold for type-I ELMy H-mode aiming at a value of $2.0<\beta_{N}<2.5$, and is then kept for a significant part of a current diffusion time before the power is ramped up further to obtain the highest $\beta_{N^{-}}$ value, limited to $\beta_{N} \approx 3.0-3.5$ by the onset of $(2,1)$-neoclassical-tearing-modes (NTMs). The latter high- $\beta$ phase is envisaged for the ITER hybrid scenario. The phase with constant intermediate power turns out to be important to prevent an early onset of strong NTMs in the final high power phase. The phenomenological dependence of the plasma state on time scales 
in the order of the current diffusion time indicates that the current profile is an important ingredient in the process. Still, the underlying physics of the formation process of the hybrid scenario is not well understood. A central element in the discussion is the suppression of sawteeth allowing higher beta-values by elimination of seed-islands for detrimental NTMs. Still small amplitude NTMs as well as small amplitude sawteeth are found in some of the high- $\beta$ phases without detrimental effects. Results from DIII-D [2] show an anti-correlation between the amplitude of the $(3,2)$-NTM and the amplitude of the sawteeth. The presence of a $(3,2)$-NTM can either reduce the size of the sawteeth such that they are less effective as a seed for a $(2,1)$-NTM or even fully stabilise the sawteeth. These findings indicate that the central q-profile is modified in presence of NTMs. Still there are open questions on why the NTM is created and why it stays small and how this relates to the heating in the ramp-up phase. Another observation still lacking explanation is the small power degradation of the energy confinement time with increasing heating power, such that the high $\beta$-values are achieved with $\mathrm{H}$-factors well above unity (referring to the ITER reference H-mode scaling IPB98(y,2) [8]). Here core and pedestal effects still have to be separated.

Recent studies at ASDEX Upgrade on the improved H-mode/ITER-hybrid-scenario are published in a series of papers: The performance of the strong heating phase (hybrid-phase) over a wide operational range, its dependence on machine conditions, and its extrapolation to ITER are described in [9]. The high resolution pedestal measurements and details of the pedestal evolution with increasing heating power are described in $[10,11]$. These are compared to the findings of other machines on the pedestal behaviour in improved confinement discharges in [12].

This paper addresses effects of the ramp-up phase on the current profile in the main heating phase and the consequences for plasma performance. In section 2 the systematic variations of the ramp-up phase are described. The effects on the current profile and observed MHD are described in section 3 and are compared to earlier results of ASDEX Upgrade in section 4. The underlying physics which relates the observed changes of the kinetic profiles to the observed changes of the current profile and the MHD are discussed in section 5. A 
concluding section closes the paper.

\section{Variation of the ramp-up scenario}

The current ramp-up phase is thought to be crucial for improved H-mode operation in the sense of sawtooth prevention. Early additional heating in the current ramp-up increases the electron temperature and therefore the current diffusion time, such that the loop voltage applied by the central solenoid penetrates less towards the plasma centre and the resulting plasma-current profile at the start of the flat-top phase is less peaked or even hollow keeping the central safety factor well above unity. On the other hand, too much power in the preheat is known to generate in the subsequent main heating phase a short-lived internal transport barrier (ITB) in the ion channel followed by a plasma collapse. However, the optimum shape of the q-profile is not obvious and usually it is determined in a trial and error procedure, such that the ramp-up scenario varies between machines. In ASDEX Upgrade the ramp-up scheme had to be changed after the inner wall was coated with tungsten in 2003 . Prior to this, the inner wall was used as high-field-side limiter during the whole ramp-up phase. Afterwards, the change-over to a diverted shape was timed shortly after the NBI is switched on. The usual criterion to accept a ramp-up scenario as reasonably optimised is that a type-I ELMy H-mode with no or modest sawteeth evolves and that high $\beta$-values are obtained in the high-power hybrid-phase. It was therefore surprising to observe in ASDEX Upgrade that an explicit late heating scheme with a long ohmic phase before adding auxiliary heating resulted in a better performance (higher stored energy $W_{m h d}$ and higher $\mathrm{H}$-factor) as compared to the dedicated early heating scheme. The first observations of this behaviour are shown in figure 1 [11]. The usual early-heating scenario (left) is compared to the late-heating scenario (right) for three levels of heating power during the flat top of the plasma current. Especially for the first level ( $8 \mathrm{MW}$ of additional heating) the stored energy is about $20 \%$ higher for the late heating scenario. This is not due to an increased fast particle content, since the the NBI-beams are the same and the plasma density is even larger. This result obviously questions the above mentioned ideas on the effect of the current ramp-up phase. Figure 1 also shows that at the 
highest power level $(\approx 13 \mathrm{MW})$ a similar stored energy is obtained $\left(\beta_{N} \approx 3\right)$ independent from the ramp-up scenario, which means that for this phase also the energy confinement is similar. During this phase a (3,2)-NTM was also present for the late-heating scenario, which is present for the early-heating scenario from $1.7 \mathrm{sec}$ onwards.

To analyse these surprising findings in more detail, a systematic study of the early heating phase was performed varying heating power and gas puff at two different values of the toroidal field (2.0 T and $2.4 \mathrm{~T}$, corresponding to $q_{95}$ values of 4.0 and 4.8 , see table 1 for details). The results were compared to the late heating scheme, also at both values of $q_{95}$. The main heating phase was kept at a constant level of $8 \mathrm{MW}$ for all discharges including $3 \mathrm{MW}$ of central ICRH. Central ICRH is necessary to prevent central accumulation of high-Z impurities in this scenario, after large parts of the first wall of ASDEX Upgrade have been covered with tungsten [9]. Since stray radiation from the ICRH-plant is suspected to disturb the MSE measurement, ICRH is switched off $50 \mathrm{~ms}$ out of $500 \mathrm{~ms}$ during the discharge and is substituted by another NBI source. The choice of NBI sources was the same in all discharges, such that profiles of the NBI driven current and momentum input are similar. Co-injecting NBI sources are used: one beam, which is also used in the current ramp-up, passes through the plasma centre (i.e. on-axis), whereas another one is off-axis [13]. A third beam similar to the first one is used to substitute the ICRH during the short intervals of MSE measurements. Due to the variation of the energy confinement time $\tau_{E}$ and the toroidal field $B_{t}, \beta_{N}$ ranges from 2.0 to 2.8 in these discharges. It was deliberately chosen not to increase the heating power to the $\beta$-limit towards the end of the discharge, but to let the current profile completely evolve. The objective was to check whether the current profiles which we observe in the main heating phase are really separate (meta)-stable states or if they evolve towards a common equilibrium with similar MHD-modes. Figure 2 illustrates the two ramp-up schemes by time traces for an early-heated and a late-heated discharge at $q_{95}=4.8$. 


\section{Effect of ramp-up variations on current profile and energy confinement}

Figure 3 shows the q-profiles which have been achieved at the onset of the main heating phase due to the variation of the start-up scenario (i.e. at $1.0 \mathrm{~s}$ for the early heating and at $2.5 \mathrm{~s}$ for the late heating). All discharges have otherwise identical control parameters, i.e. 1.0 MA plasma current, same shape, no additional fuelling during the main heating phase. Table 1 lists the modifications during current ramp-up, the achieved values for $q_{0}$ at the onset of the main heating, $\mathrm{H}$-factors and MHD-modes in the equilibrated phase towards the end of the main heating. With early heating the $q$-profile is usually inverted towards the plasma centre whereas for late heating it is monotonic with $q_{0}$ close to unity. Not surprisingly an increase of the gas puff during ramp-up does reduce the inversion of the current profile in the early heated case. The evolution of the $q$-profile during the main heating phase is shown in figure 4 for the discharge with the most strongly inverted $q$-profile at the onset of the main heating. The major changes are observed for the first half second but a steady state is only reached after 3 seconds of flat top. During this evolution of the current profile, a (4,3)-NTM occurs at 2 seconds, followed by the occurrence of a $(3,2)-\mathrm{NTM}$ at 3.2 seconds.

Figure 5 shows that the equilibrated q-profiles are only marginally different for all $q_{95}=4.8$ cases with early heating, essentially independent from the variations of heating power and gas puff in the preheat phase. The case with strongest gas puff (\#20990) shows small sawteeth, but no difference with respect to confinement or other MHD activity is observed. Comparing the blue curves in figures 3 and 5 we note, that the current profile obtained with the late-heating scheme remains almost unchanged in time (from $2.5 \mathrm{sec}$ onwards), i.e. it is already close to its equilibrated shape.

As shown in table 1, there was only one ramp-up scenario for which no steady state $\mathrm{H}$ mode was obtained during the main heating phase: for $q_{95}=4.0$ the early heating scenario with the highest level of early heating developed a strong ion ITB followed by a disruption, as is usually observed for stronger heating during current ramp-up. The reason for this different behaviour is an L-H transition (due to the reduced $B_{t}$ ) already in the current ramp-up phase, 
leading already in this phase to $T_{i}$ values well above $T_{e}$. Such a target plasma is especially prone to develop an ion ITB with additional NBI heating. We refer to [14], in which the dilution of the thermal ions by the fast ion population produced by the NBI in low density plasmas is identified as a key element to generate an ion ITB on ASDEX Upgrade due to stabilisation of ITG-modes.

The first discharge at lower $q_{95}$ showed a significant sensitivity to the periodic switching of ICRH and NBI. Therefore, this switching has been turned off for the other discharges at this q-value, as such no MSE values are available. In the following, we therefore first focus on the $q_{95}=4.8$ case and will compare the MHD-modes and confinement of the lower $q_{95}$ case in section 5.4.

As can be seen from table 1 the early and late heating cases are most prominently separated by a $20 \%$ higher $\mathrm{H}$-factor (and stored energy) in the late heating case as well as by the q-profiles at the onset of the main heating. The differences of the equilibrated qprofiles at the end of the main heating phase are much smaller (figure 5) and an error analysis is required to decide whether these differences can be regarded as significant. Figure 6 shows the q-profiles for both cases including local error-bars at the location of the MSE channels [15]. These error bars overlap over the whole radius, but about half of the error bar is due to uncertainties of the exact shape of the flux surfaces. Since we are dealing with almost identical plasmas, the geometrical error must be largely correlated for both curves and does not need to be considered when deciding if the curves are different with respect to the error bars. With this argument we conclude that these two equilibrated q-profiles are significantly different for $0.3<\rho_{\text {tor }}<0.6$. An additional difference is the MHD-behaviour: the early heating scheme triggers early $(4,3)$ - or $(3,2)$-NTMs, whereas the late heating scheme shows only (1,1)-fishbones. We note here that both types of modes have already been discussed in literature as possible mechanism for modifying the current profile evolution. As mentioned above, $(3,2)-N T M s$ in hybrid-discharges in DIII-D are discussed in [2]. For fishbones we refer here to [16] analysing the first improved H-modes obtained in ASDEX Upgrade, which were dominated by fishbones. 


\section{Current ramp-up phase: limiter versus divertor configuration}

As mentioned above, the first improved H-modes observed in ASDEX Upgrade [1] also showed fishbones during the high confinement phase. For these discharges the early heating scheme was used, in fact the ramp-up of these discharges was a result of an empirical development towards improved operation. These early-heated improved H-modes with fishbones are similar in performance to the plasmas with fishbones recently obtained with the late-heating scheme as shown in figure 7. Both discharges shown in figure 7 were run without gas puff in the flat-top phase. The significant difference in the density (natural density) is most likely due to the tungsten coverage of the plasma facing components which do release a significant amount of adsorbed Deuterium when in contact with the plasma.

The blue curves in figures 7 and 2 correspond both to the new late-heating scenario. Comparing both figures clearly shows that the performance of the old early-heating scenario (figure 7) was significantly better than the performance of the early-heating scheme presently used (figure 2). These contradicting results for the older and the present early heating scheme can be resolved by analysing the differences between the two. As already mentioned above, the plasma configuration during the ramp-up had to be modified in order to cope with the increasing $\mathrm{W}$-coverage. The transition to a divertor configuration was moved to an earlier time point, i.e. from $1.0 \mathrm{~s}$ to $0.45 \mathrm{~s}$. This means that in the old scenario the plasma was in a limiter configuration during the whole current ramp-up, whereas in the new scenario the plasma was in a divertor configuration during the current ramp-up, at least for most of the time when additional heating is applied (For details on the evolution of shape and timing of the heating see [9]). Therefore the electron temperature profiles in the preheating phase are significantly different, as shown in figure 8. With the older limiter ramp-up configuration the $T_{e}$-profiles were significantly more peaked $\left(T_{e}(0) / T_{e}(0.8)\right.$ is about a factor 2 larger) as compared to the diverted ramp-up used presently. This leads to a higher current density and a lower $q$-value on axis, which may be the reason that $(1,1)$-fishbones appeared under these conditions instead of $(4,3)$ or $(3,2)$ NTMs as in the case of the present diverted ramp-up 
configuration. Unfortunately, a one-to-one comparison of old and new MSE data is impossible due to modifications of the diagnostic. There are no clear indications for an $\mathrm{L} / \mathrm{H}$-transition in the diverted configuration such that we have to ascribe the differences of the $T_{e}$-profile essentially to the strong cooling of the edge by the limiter.

These findings show that the new early-heating scheme is inferior to the old one due to too slow current penetration for $q_{95}>4.0$ (for which the old scheme has been run). This is not necessarily the case for lower values of $q_{95}$ for which a slower current penetration may be beneficial to adjust $q_{0}$ close to 1.0 at the onset of the main heating as further discussed in section 5.4.

\section{Effects of MHD and related current profile on confinement}

The different confinement properties of the H-modes obtained with the present early and late heating scheme (fig. 2) may be a direct consequence of the different MHD-modes and the related differences in the current profile (fig. 6). As possible mechanisms one can either postulate a direct influence of the NTM by short-cutting magnetic heat insulation across the island width or of effects of the modified current profile on core and/or pedestal transport. For the pair of discharges shown in figure 2 all three effects may play a role. Figure 9 shows the density profiles of the respective discharges, fitted to far-infrared interferometry plus Lithiumbeam-diagnostic. The difference is most significant at the pedestal top. Electron and ion

temperatures are shown in figures 10 and 11, respectively. We note that the temperature values close to the pedestal top are similar in both cases but the gradients in the confinement zone are larger for the late-heating case.

\subsection{Direct NTM Effect}

The ECE-data (figure 10) show that the electron temperature profiles of both discharges are significantly different only inside $\rho_{\text {tor }} \approx 0.5$. The ECE-data show a flattening of the $T_{e^{-}}$ profile just where the equilibrium reconstruction (including MSE) yields the $q=1.5$ surfaces, i.e. a flattening due to the $(3,2)$-island. Since the gradient length further inwards usually is 
limited by the nature of the underlying transport, the flattening of the $T_{e}$-profile at $q=1.5$ also reduces $T_{e}$ inside the resonant q-surface.

\subsection{Current profile effects $L_{T i}$-threshold for ITGs}

The CXRS ion temperature data (figure 11) show that the differences between the two $T_{i^{-}}$ profiles extend over a wide radial range. This variation of the gradients over a wide radial range cannot be ascribed to the (3,2)-island alone, but requires a different explanation. First we note that the variation of the gradient length $L_{T i}=T_{i} / \nabla T_{i}$ is of the order of $20 \%$ (see also figure 13 below) and the absolute values for both cases do not exceed the range of variation usually found for H-modes. This indicates that also for these improved $\mathrm{H}$-mode plasmas the temperature gradient length is limited by the destabilisation of Ion-Temperature-Gradient (ITG) modes above a threshold value of $\nabla T / T$, as reported already in [19]. Still figure 11 shows that there is an experimentally significant difference between the $T_{i}$-profiles obtained with the early-heating and the late-heating scheme. Since we have reliable q-profiles for these cases, we can calculate the ratio of magnetic shear and safety factor, i.e. $s / q$, known to influence the critical temperature gradient length for ITG turbulence [20]. To determine the shear values from the $q$-profile and the poloidal-flux matrix we use $s(\rho)=2 \frac{V(\rho)}{q(\rho)} \frac{d q}{d V}(\rho)[21]$, where $\mathrm{V}$ is the flux surface volume. For circular plasmas this simplifies to the usual definition $\frac{r}{q} \frac{d q}{d r}$, since $\frac{d V}{d r}=2 \frac{V}{r}$ in this case. Figure 12 compares the profiles of $s / q$ for both scenarios and the solid lines in figure 13 are the $R / L_{T i}$ profiles obtained from the fits to the experimental $T_{i}$ data. In the same figure, the threshold values of $R / L_{T i}$ for the linear onset of the ITG mode at mid-radius for the two plasmas are shown. These have been computed with GS2 [22] as the values of $R / L_{T i}$ at which the maximum growth rate is equal to the value of the $E \times B$ shearing rate $\omega_{E \times B}[23]$. The calculations are made in such a way to quantify exclusively the effect due to the variation of the shear and safety factor profiles in the two plasmas. This implies that for both scenarios the $E \times B$ shearing rates are fixed to the experimental value of the late-heating scenario, which is somewhat higher than the experimental value of the early-heating scenario. In these gyrokinetic calculations an s- $\alpha$ equilibrium is used. Comparing both discharges we 
note that the difference of the calculated threshold values is close to that of the measured values. This shows that the theoretically predicted effects on ion heat transport due to the observed changes in the current profile are large enough to cause changes of the $T_{i}$ profiles which are in the observed range.

\subsection{Variation of the H-mode pedestal}

As mentioned above, also the pedestal pressure is higher in the late-heating case due to increased pedestal density. It is well known that the plasma current in the plasma edge has a strong influence on pedestal behaviour and therefore modifications of the current profile could in principle influence the pedestal. Unfortunately, for these discharges we do not have high-resolution Thomson-scattering measurements at the plasma edge, which are necessary to resolve the steep gradients of density and temperature as needed for a proper reconstruction of the bootstrap current in the edge. Such high-resolution Thomson-scattering data were obtained under these conditions for a pair of discharges from the previous campaign (similar to figure 1). In [11] analysis of the plasma edge is reported. With respect to the density profile this analysis shows that the width of the steep density-gradient zone is similar for the earlyand the late-heating scenario but steeper density gradients in the H-mode pedestal and a higher separatrix density lead to higher pedestal densities in the late-heating case. Unfortunately, these discharges with good edge measurements did not have proper MSE-data, such that also for this pair it is not possible to link central current profile and edge current profile. A recent comparison of these results with pedestal measurements on DIII-D, JET and JT-60U is described in [12].

\subsection{Results at lower $q_{95}$}

In table 1 it is shown that this study also included discharges at $q_{95}=4.0$, whereas for the discharges discussed above in detail the value of $q_{95}$ was 4.8. As mentioned above, the MHD behaviour during the first discharge at lower $q_{95}$ showed clear correlations with the modulation of ICRH and NBI applied to obtain useful MSE measurements. Therefore, this modulation 
has been turned off for all subsequent discharges at this lower $q_{95}$ value and no $q$-profiles are availabe for these discharges during the main heating phase during which the current-profile equilibrates. The equilibrated $q$-profile obtained from the first attempt (\#20996), as shown in figure 5 may be influenced by the modulation.

Still several clear observations have been made as well for $q_{95}=4.0$, as shown in table 1 . As for $q_{95}=4.8$ the late heating scheme leads to fishbone dominated plasmas with an H-factor around 1.5 whereas the early heating scheme yields NTM dominated plasmas with an H-factor close to 1.2. These findings at least suggest that the underlying physics is similar for both values of $q_{95}$. Figure 14 shows that for $q_{95}=4.0$ it is more difficult to achieve the fishbone dominated regime with the late heating scheme. During the first two seconds of the main heating phase the (4,3)-NTM dominates, but short phases with fishbones are also present. These phases increase in length and at $4.5 \mathrm{sec}$ the NTM changes from $(4,3)$ to $(5,4)$ and the fishbone amplitude increases significantly. During these first 2 seconds of the main heating the $\mathrm{H}$-factor rises from 1.3 to 1.5 . In contrast, the late heated discharges with $q_{95}=4.8$ did not show any NTMs before the fishbones developed. The reason for the different behaviour may be linked to the central $q$-value at the onset of the main heating. As shown in table 1 and figure 3 it is well below 1.0 for $q_{95}=4.0$ preventing an early appearance of fishbones. This indicates that the late heating scheme can generate the favourable fishbone dominated $\mathrm{H}$ modes only above a minimum value of $q_{95}$ close to 4.0. For significantly lower values of $q_{95}$ a slower current penetration during the ramp-up phase is necessary to achieve a central $q$-value close to 1.0 at the onset of the main heating. This could be obtained by an optimised early heating scheme. Indeed, using the present early heating scheme, high-performance improved $\mathrm{H}$-modes are obtained at $q_{95}=3.1$ dominated by fishbones [9]. These discharges are similar to the $q_{95}=4.0$ case discussed above except that the plasma current is $1.2 \mathrm{MA}$ instead of $1.0 \mathrm{MA}$. For these higher currents, measurements of the current profile as well as a comparison with the late heating scheme are still to be done. 


\section{Discussion and Conclusions}

In the previous section it was described that, for $q_{95} \geq 4.0$, significantly different equilibrated current profiles can be obtained, depending on the ramp-up scenario. The energy confinement is significantly differnt as well. The specific early heating scenario had poorer energy confinement than the late heating scenario most likely due to a combination of the presence of NTMs and the related $q$-profile modifications. This correlation can occasionally be observed also in a single flattop phase during which the $(4,3)$ or $(3,2)$ NTM appears or disappears: The disappearance of a $(4,3)$-NTM coincident with increasing confinement has been described at the end of the previous section for a $q_{95}=4.0$ discharge of our series (figure 14). Vice versa, in [24] an early heated improved H-mode is shown, for which the $(3,2)$-mode does set in well after the start of the main heating. At first, the main heating phase showed only a weak $(5,4)$ NTM. Immediately after the onset of the $(3,2)$-mode a reduction of the stored energy of more than $10 \%$ is observed.

The maximum achievable value of $\beta$ seems to be very similar for NTM stabilized qprofiles and fishbone stabilized q-profiles. The discharge shown in figure 14 reaches $\beta_{N}=2.8$ in the phase with fishbones, examples in [9] reach $\beta_{N}=3.0$ with fishbones but no NTM.

From the $q$-profile evolution shown in figure 4 it seems that in the early-heated discharges the NTM sets in as the q-profile drops to the corresponding q-values. As the central q-profile is very flat at that time point, the shear in the region close to the respective rational $q$-value is close to zero, which facilitates the formation of the NTM. For the late heating case, the central $q$-value is already close to unity favouring the occurence of fishbones. In this case the shear at $q=1.33 \cdots 1.5$ is significant, possibly stabilising the NTMs although $\beta$ is higher.

In terms of a start-up recipe these results suggest that for the onset of the main heating a flat central $q$-profile with $q \approx 1$ should be aimed at. In ASDEX Upgrade this was possible with an ohmic plasma ramped-up in a divertor configuration, but most likely also with early heating of a plasma ramped-up in a limiter configuration. There may be other ways, especially with dedicated tools such as LHCD [4] or ECCD to modify the current profile before the onset 
of the main heating or during the main heating. However, the use of these tools may require subtle adjustments: DIII-D experiments on stabilisation of the (3,2)-mode with ECCD [2] resulted in larger sawteeth and no fishbones were reported. Seemingly, the $q$-profile on axis dropped well below unity, indicating that it may be necessary to counteract the full relaxation of the $q$-profile when stabilising the NTM. On ASDEX Upgrade it was possible to achieve sawtooth-free fishbone-dominated high $\beta_{N}$ operation at $q_{95} \approx 3$ after stabilising a (3,2)-NTM [9]. As mentioned above, the NBI driven current may be important as well for the $q$-profile evolution, therefore it was kept constant in this study. Nonetheless, it was different in the first fishbone-dominated improved H-modes in ASDEX Upgrade [1]. An extension of this study including variation of the beam sources is planned, although previous experiments showed that the $q$-profile is hardly modified by off-axis NBCD in strongly heated H-modes [25]. Understanding the mechanisms by which the MHD-modes prevent the full relaxation of the current profile remains an open question. Such an understanding is essential to predict if these mechanisms can be effective also in bigger machines such as ITER. Similarily, a higher level of understanding is necessary to predict if fishbone-modes will form in ITER, since they do not only require a flat q-profile close to 1 , but also a sufficient fast particle drive. Lacking so far the necessary physics understanding, it is at least encouraging that also in a larger device, such as JET, high- $\beta$ H-modes with H-factors above unity have been observed with $q_{0} \approx 1$ showing strong fishbones [4] and NTM activity with varying amplitude and mode number.

\section{References}

[1] GRUBER, O. et al., Phys. Rev. Lett. 83 (1999) 1787.

[2] WADE, M. R. et al., Nucl. Fusion 45 (2005) 407.

[3] IDE, S. and THE JT-60 TEAM, Nucl. Fusion 45 (2005) S48.

[4] JOFFRIN, E. et al., Nucl. Fusion 45 (2005) 626.

[5] SHIMOMURA, Y. et al., Plasma Phys. Controlled Fusion 43 (2001) A385.

[6] A.C.C. Sips for the Steady State Operation and the Transport Physics topical groups of the International Tokamak Physics Activity, Plasma Phys. Controlled Fusion 47 (2005) A19.

[7] GRUBER, O. et al., Plasma Phys. Controlled Fusion 47 (2005) B135. 
[8] ITER Physics Basis, Nucl. Fusion 39 (1999) 2204.

[9] SIPS, A. C. C. et al., submitted to Nucl. Fusion, based on contribution EX 1-1: The Performance of Improved H-modes at ASDEX Upgrade and Projection to ITER.

[10] SUTTROP, W. et al., Studies of the edge pedestal and behaviour of edge localised modes in improved Hmodes in ASDEX Upgrade, in Fusion Energy 2006 (Proc. 21st. Int. Conf., Chengdu, 2006), pages CDROM file: papers/ex_p8-5 and http://www-naweb.iaea.org/napc/physics/FEC/FEC2006/papers/ex p85, Vienna, 2007, IAEA.

[11] HORTON, L. D. et al., Edge transport barrier characteristics of improved H-modes in ASDEX Upgrade, in Europhysics Conference Abstracts (CD-ROM, Proc. of the 33th EPS Conference on Controlled Fusion and Plasma Physics, Rome, 2006), pages P-2.140, Geneva, 2006, EPS, journal paper in progress.

[12] MAGGI, C. F. et al., accepted by Nucl. Fusion, based on contribution IT P1-6: Characteristics of the H-mode Pedestal in Improved Confinement Scenarios in ASDEX Upgrade, DIII-D, JET and JT-60U.

[13] SIPS, A. C. C. et al., Plasma Phys. Controlled Fusion 44 (2002) B69.

[14] TARDINI, G. et al., Ion ITB dynamics in ASDEX Upgrade, in Europhysics Conference Abstracts (CDROM, Proc. of the 32nd EPS Conference on Plasma Physics, Tarragona, 2005), edited by HIDALGO, C. and van Milligen, B. P., volume 29C, pages P-2.028, Geneva, 2005, EPS.

[15] MC CARTHY, P. J. et al., Plasma geometry and current profile identification on ASDEX UPGRADE using an integrated equilibrium generation and interpretation system, in Fusion Energy 2006 (Proc. 21st. Int. Conf., Chengdu, 2006), pages CD-ROM file: papers/thp3-7 and http://wwwnaweb.iaea.org/napc/physics/FEC/FEC2006/papers/th_p3-7.pdf, Vienna, 2007, IAEA.

[16] GÜNTER, S. et al., Nucl. Fusion 39 (1999) 1535.

[17] STÄBLER, A. et al., The role of neutral beam injection geometry in advanced discharge scenarios on ASDEX Upgrade, in Europhysics Conference Abstracts (CD-ROM, Proc. of the 29th EPS Conference on Plasma Physics and Controlled Fusion, Montreux, 2002), edited by BEHN, R. and VARANDAS, C., volume 26B, page O5.03, Geneva, 2002, EPS.

[18] HORTON, L. D. et al., Nucl. Fusion 45 (2005) 856.

[19] PEETERS, A. G. et al., Nucl. Fusion 42 (2002) 1376.

[20] GUO, S. C. and ROMANELLI, F., Phys. Fluids B 5 (1993) 520.

[21] TAKAHASHI, H. et al., Nucl. Fusion 32 (1992) 815.

[22] KOTSCHENREUTHER, M. et al., Comput. Phys. Commun. 88 (1995) 128.

[23] WALTZ, R. et al., Phys. Plasmas 1 (1994) 2229.

[24] NA, Y.-S. et al., Nucl. Fusion 46 (2006) 232.

[25] GÜNTER, S. et al., Current profile modification by off-axis NBI on ASDEX Upgrade, in Europhysics Conference Abstracts (CD-ROM, Proc. of the 32nd EPS Conference on Plasma Physics, Tarragona, 
2005), edited by HIDALGO, C. and van Milligen, B. P., volume 29C, pages P-4.075, Geneva, 2005, EPS. 

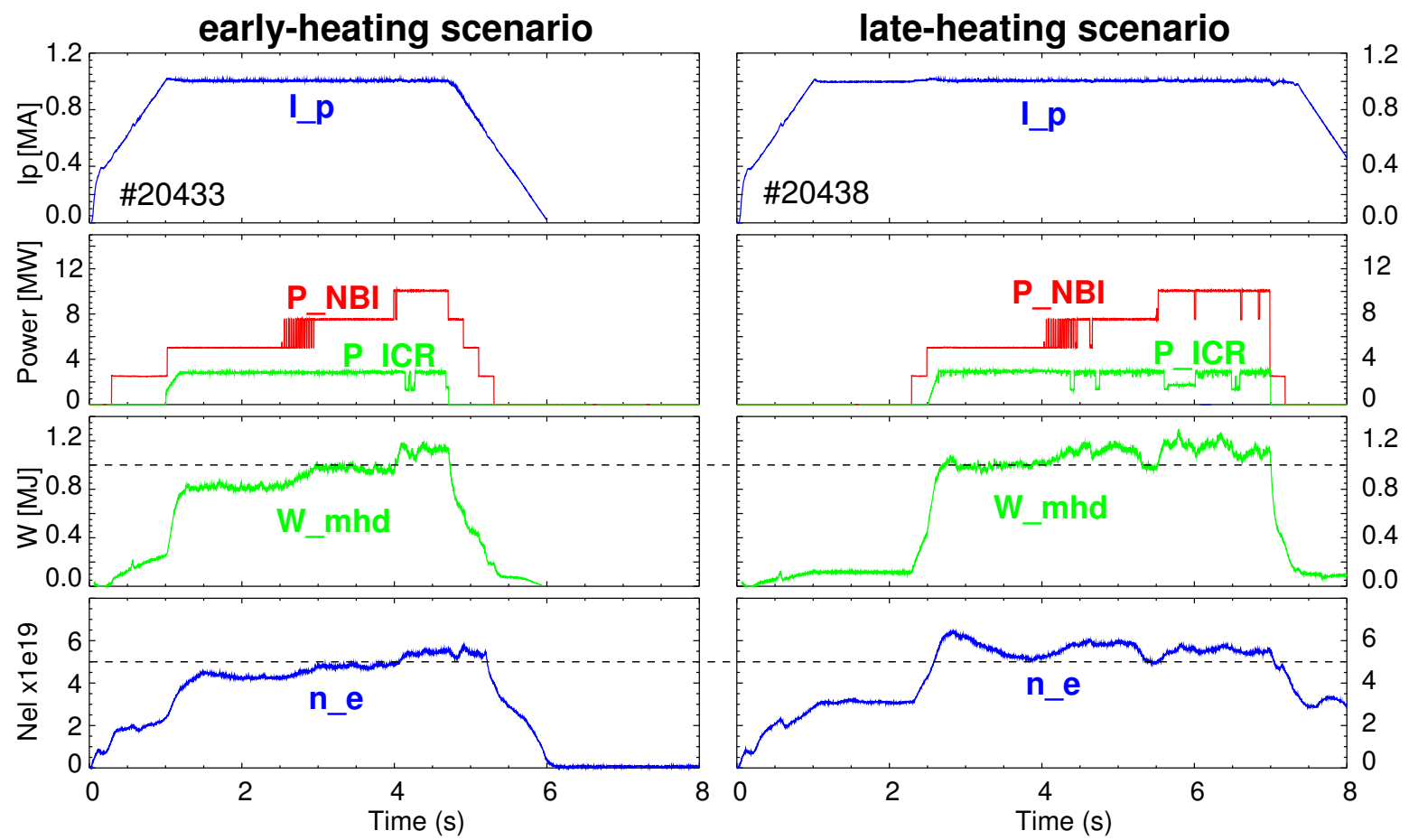

Figure 1. Effect of two different ramp-up scenarios on improved H-mode performance at different power levels. $B_{t}=2.4 \mathrm{~T}, q_{95}=4.8$, identical plasma shape and NBI geometry. The drops in the time traces of stored energy and density for the late heating scenario correspond to the onset of $(3,2)-N T M s$. With the early-heating scenario these are present from $1.7 \mathrm{sec}$ onwards. 


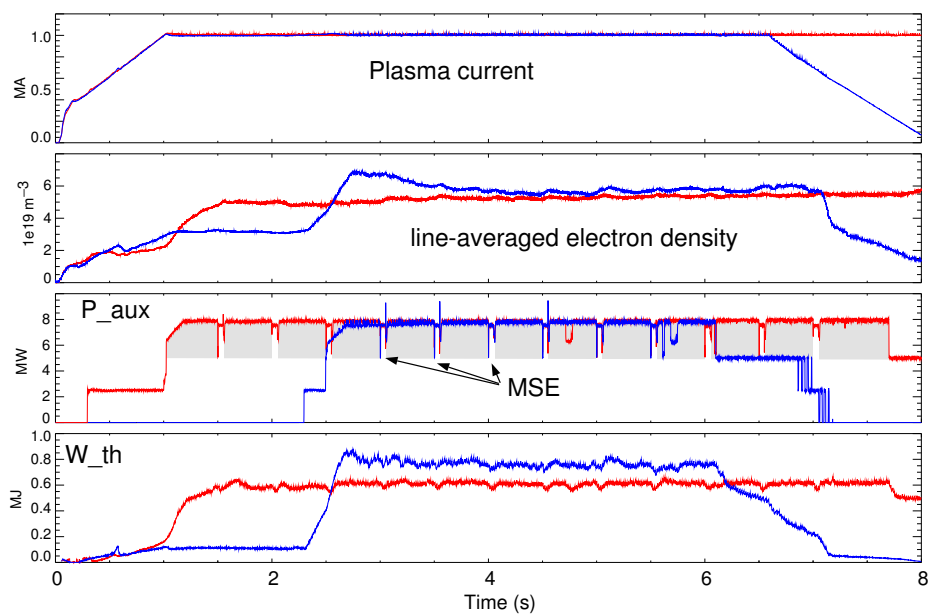

Figure 2. Time traces for the current ramp up studies corresponding to table 1. Comparison of an early-heating ramp-up (\#20993) with a late-heating ramp-up (\#20995). Both discharges have identical control parameters during the flat-top phase: $I_{p}=1.0 \mathrm{MA}, P_{a u x} \approx 8 \mathrm{MW}$, no gas puff, same shape. Still the stored energy is significantly higher for the late-heating case. Since operational parameter are almost identical this is reflected in the H-factors, which are 1.2 and 1.5 using the IPB98(y,2) scaling. The MHD-modes observed during the flat-top phase are different in the two cases: for the early-heating a (4,3)-NTM is observed at $1.8 \mathrm{~s}$ which changes to a $(3,2)+(5,4)$ NTM at $3.2 \mathrm{sec}$. The late-heating case shows $(1,1)$-fishbones during the whole phase with full heating power. The grey shaded area indicates the level of ICRH which was used to prevent $\mathrm{W}$-accumulation in the plasma centre (see text). Since the ICRH disturbed the MSE-measurement, it was switched-off for $50 \mathrm{~ms}$ every $500 \mathrm{~ms}$. During these $50 \mathrm{~ms}$ without ICRH another NBI beam was used to keep the total power constant. Therefore the time-traces of the total power show some spikes (duration $<2 \mathrm{~ms}$ ) due to imperfect synchronisation of NBI and ICRH switching. 


\begin{tabular}{|l|l|l|l|l|l|l|l|}
\hline \#shot & $\begin{array}{l}\mathrm{Te} 0 \\
(1.0 \mathrm{~s})\end{array}$ & $\begin{array}{l}\langle n e\rangle \\
(1.0 \mathrm{~s})\end{array}$ & $\begin{array}{l}\text { PNBI } \\
(\text { preheat })\end{array}$ & $\mathrm{q} 0$ & $\mathrm{q} 95$ & $\mathrm{H} 98_{y, 2}$ & $\begin{array}{l}\text { MHD behaviour during } \\
\text { high power phase }\end{array}$ \\
\hline & {$[\mathrm{keV}]$} & $1{\mathrm{E} 19 \mathrm{~m}^{-3}}^{-3}[\mathrm{MW}]$ & & & & \\
\hline 20998 & 3.0 & 2.5 & 2.5 & 2.0 & 4.0 & - & Disrupts after ITB \\
\hline 20993 & 3.4 & 2.2 & 2.5 & 3.2 & 4.8 & 1.2 & $4 / 3$ NTM, 3/2 NTM at 3.2s \\
\hline 20991 & 2.6 & 3.6 & 2.5 & 2.8 & 4.8 & 1.2 & $4 / 3$ NTM, 3/2 NTM at $2.3 \mathrm{~s}$ \\
\hline 20990 & 2.2 & 5.5 & 2.5 & 2.2 & 4.8 & 1.2 & $4 / 3$ NTM, 3/2 NTM at $1.8 \mathrm{~s}$ \\
\hline 20992 & 1.5 & 5.3 & 1.25 & $?$ & 4.8 & 1.2 & $4 / 3$ NTM throughout \\
\hline 20994 & 1.6 & 2.9 & 0 & 1.0 & 4.8 & - & Fishbones, wall contact \\
\hline 20995 & 1.6 & 2.9 & 0 & 0.95 & 4.8 & 1.5 & Fishbones throughout \\
\hline 20996 & 1.1 & 2.9 & 0 & 0.8 & 4.0 & 1.5 & Fishbones \\
\hline 20997 & 1.1 & 2.9 & 0 & 0.8 & 4.0 & 1.5 & $4 / 3$ NTM $\rightarrow$ Fishbones \\
\hline 20999 & 1.0 & 5.2 & 1.25 & $?$ & 4.0 & 1.2 & $4 / 3$ NTM throughout \\
\hline
\end{tabular}

Table 1. Parameters of the current ramp-up studies. All discharges have some sawteeth, partly synchronised with ICRH modulation. $q_{0}$ is given at the onset of the main heating, i.e. at 1.0 $\mathrm{s}$ with preheat and at $2.5 \mathrm{~s}$ without. No reasonable MSE measurement is possible for the 1.25 MW preheat level, realized by fast modulation of the $2.5 \mathrm{MW}$ beam viewed by the MSE diagnostic. This is indicated by a '?' in the 'q0' column. The H-factor refers to the steady-state phase at $5.5 \mathrm{~s}$ in all cases. 


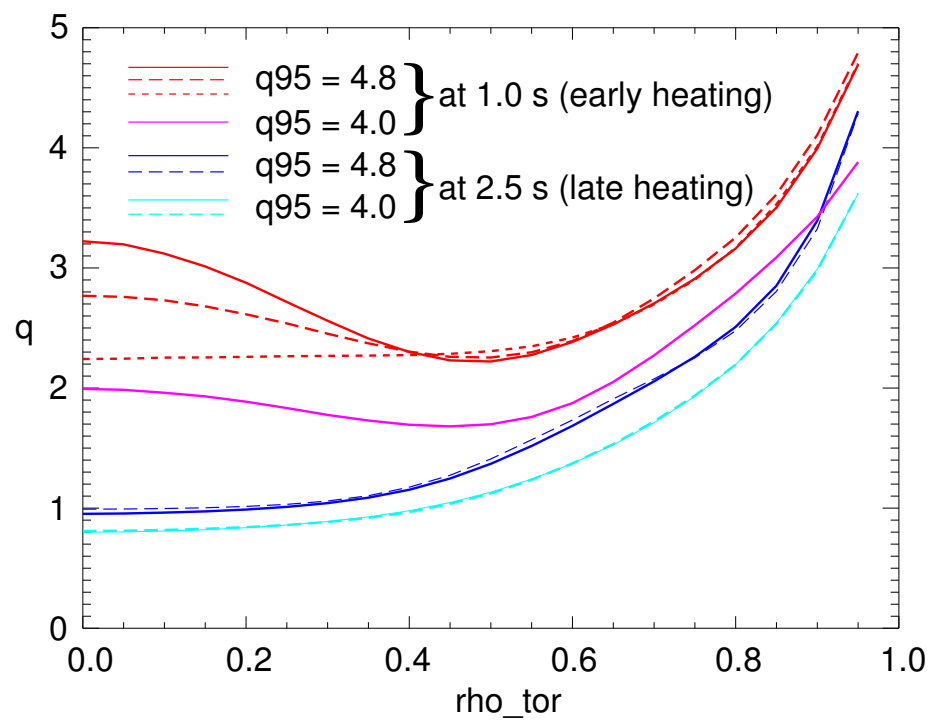

Figure 3. Variation of q-profiles at the onset of the main heating obtained by modification of the ramp-up phase. In the order of decreasing $q_{0}$ the curves are identified as follows: early heating, $q_{95}=4.8$, 3 levels of gas puff (solid:\#20993, dashed:\#20991, dotted:\#20990); early heating, $q_{95}=4.0$ (solid:\#20998); late heating, $q_{95}=4.8$ (solid:\#20995, dashed:\#20994); late heating $q_{95}=4.0$ (solid:\#20997, dashed:\#20996). The time of the q-profile measurement is identical to the time for the $q_{0}$-values given in table 1 . (Here and in the following we use the flux surface label $\rho_{t o r}=\sqrt{\Psi / \Psi \text { separatrix }} . \Psi$ is the toroidal magnetic flux through the respective surface and is zero on axis.) 


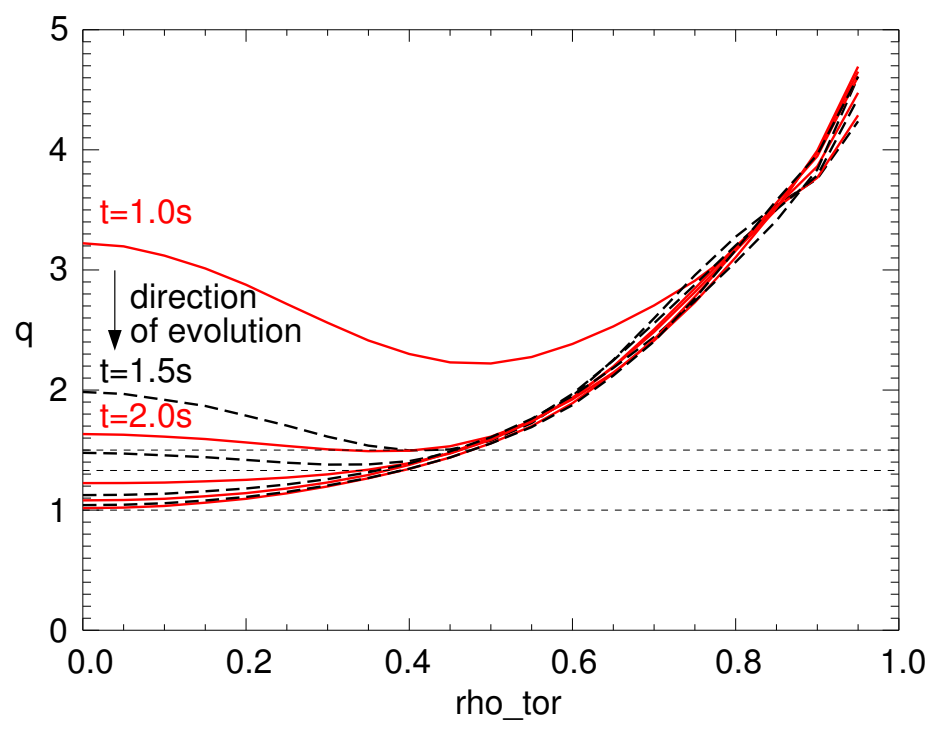

Figure 4. Evolution of the q-profile during the main heating phase for the most inverted qprofile in figure 3 (early-heating case in figure 2). The time-evolution is shown in steps of $0.5 \mathrm{~s}$ starting at $1.0 \mathrm{~s}$. The central $q_{0}$ evolves towards lower values. The three horizontal lines indicate resonant q-values (3/2, 4/3 and 1$)$.

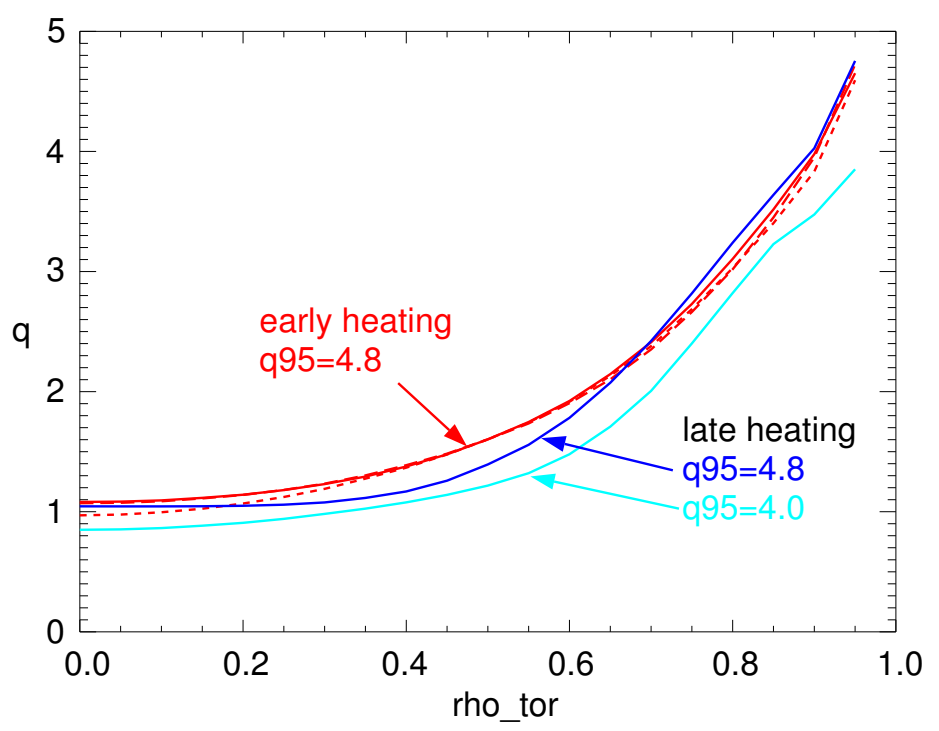

Figure 5. Equilibrated current profiles (at $5.5 \mathrm{~s}$ ), same linestyle/colour-code as in fig. 3. (if MSE is available). Error bars are discussed in the text on the basis of figure 6. Only the short dashed red case (\#20990, see table 1) shows regular sawteeth not related to ICRH modulation. 


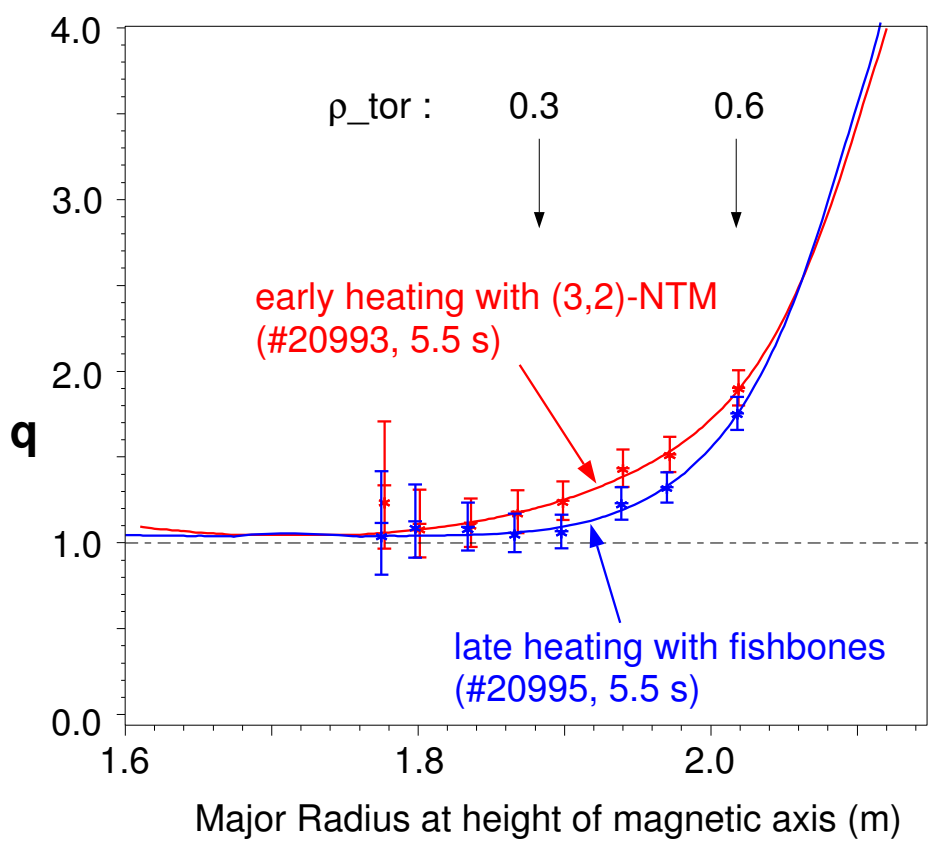

Figure 6. Local error bars of the q-profile from MSE-diagnostic for the early (red) and late (blue) heated discharges from fig. 2 towards the end of the heating phase at 5.5s. The profiles are identical to those shown with same colour and solid lines in fig. 5. The MSE data are taken close to the outer midplane. The separatrix is at $R=2.15 \mathrm{~m}$ and the plasma centre at $R=1.72 \mathrm{~m}$. 


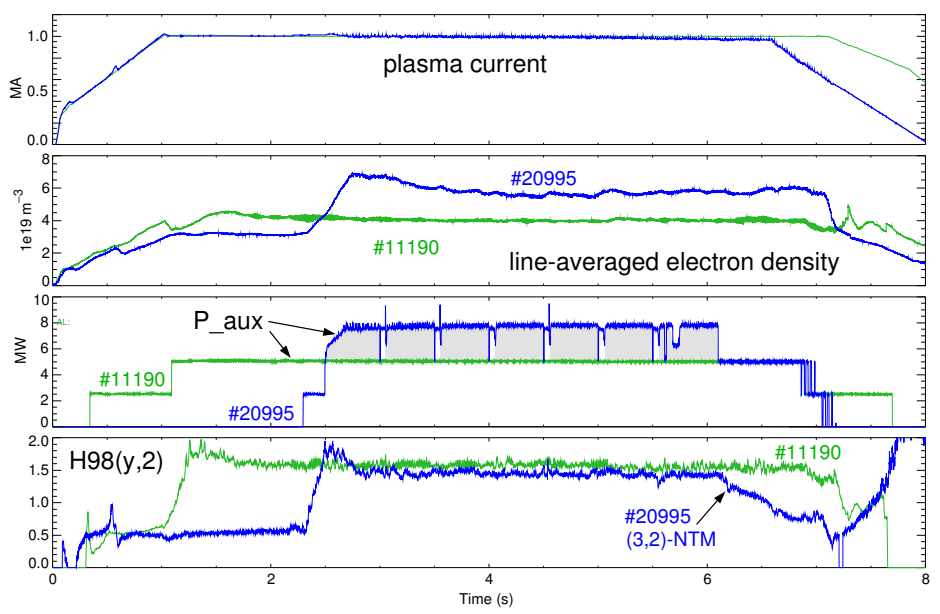

Figure 7. Time traces for one of the first improved H-modes in ASDEX Upgrade (\#11190,[1]) compared to a recent late-heated discharge (\#20995, see table 1). Plasma current and toroidal field are identical for both discharges shown here $\left(q_{95}=4.8\right)$. As in figure 2 the grey shaded area indicates applied ICRH for \#20995. The necessity of using ICRH with dominantly Wcoated plasma-facing components becomes obvious from the strong reduction in performance after switching off the ICRH at $6.1 \mathrm{~s}$, which is partly due to $(3,2)+(5,4)-\mathrm{NTMs}$ which set in at $6.2 \mathrm{~s}$, about one $\tau_{E}$ or $0.2 \times \tau_{R}$ after the ICRH is switched off. The NBI power in the flat-top phase is the same for both discharges ( 2 beams, $2.5 \mathrm{MW}$ each), but one of the beams is more co-injecting and more off-axis for $\# 20995$ and for this beam also the voltage is higher, i.e. $93 \mathrm{kV}$ instead of $60 \mathrm{kV}$ (see $[17,13]$ for modifications of the beam geometry). 


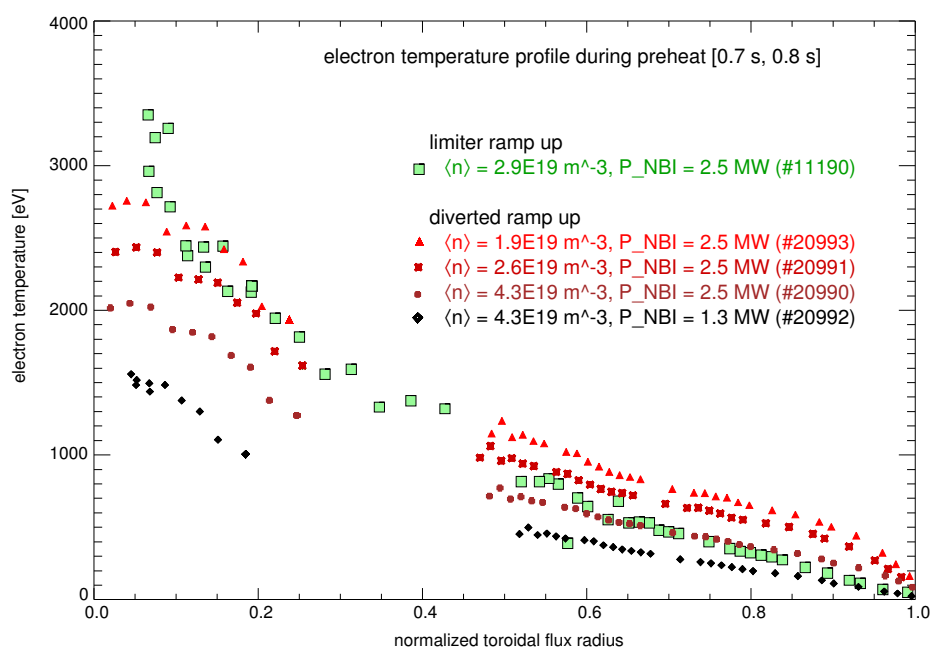

Figure 8. Electron temperature profiles during preheat (averaged between 0.7 and $0.8 \mathrm{sec}$ ). A sequence of recent early-heated discharges with variations of gas puff and NBI-power (see table 1) is compared to one of the first improved H-modes in ASDEX Upgrade (\#11190,[1], see also fig. 7). Note that for the recent discharges an intermediate range of ECE-channels was not available.

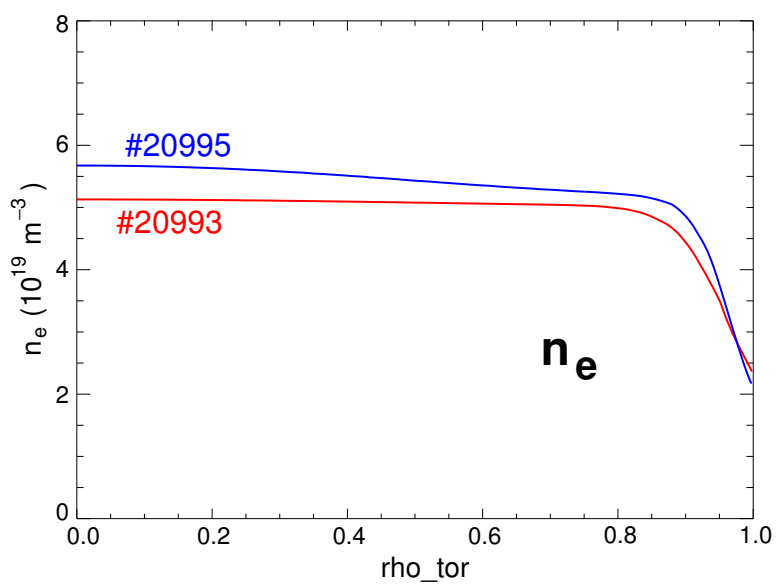

Figure 9. Electron density profiles for the early (red) and late (blue) heated discharges from figs. 2,6 (at $5.5 \mathrm{~s}$ ). The curves presented here are polynomial fits from a common evaluation of Thomson scattering, Interferometry and Li-beam measurements [18]. 


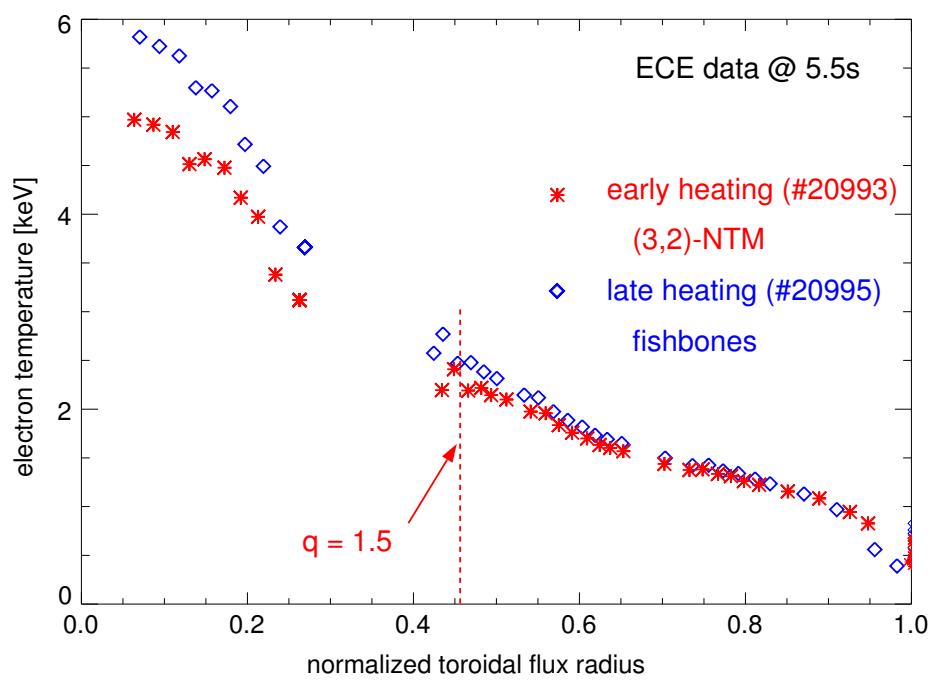

Figure 10. Comparison of $T_{e}$-data from ECE for the discharges shown in fig. 2 towards the end of the flat-top phase. The dashed vertical line indicates the radial position of the $q=1.5$ surface for discharge \#20993 (early-heating) as obtained from the equilibrium reconstruction using MSE data (figure 5).

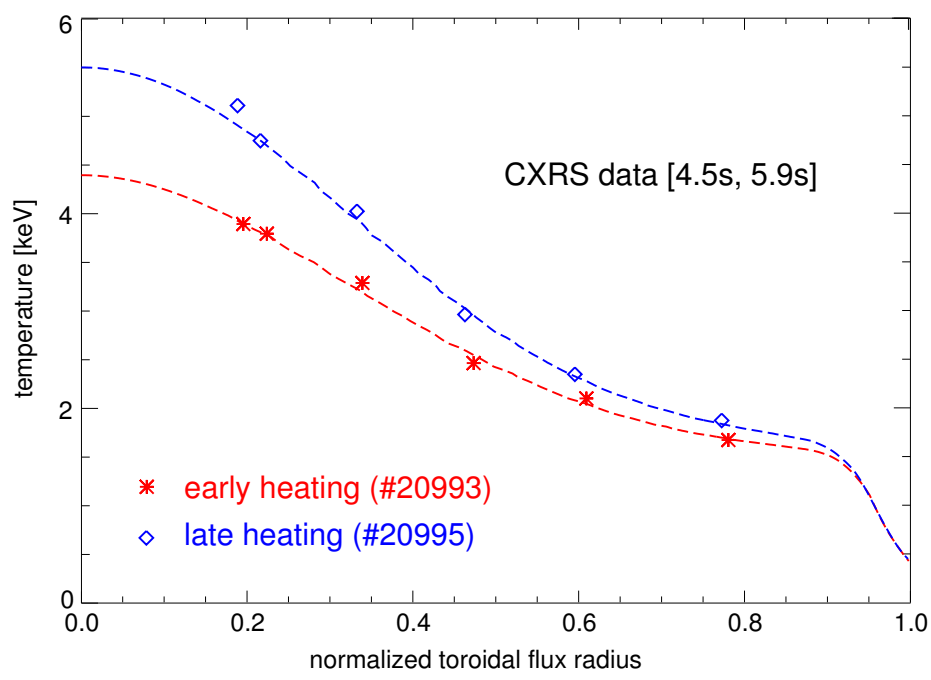

Figure 11. Comparison of $T_{i}$-data from CXRS for the discharges shown in fig. 2 towards the end of the flat-top phase. Channels are averaged in time between $4.5 \mathrm{~s}$ and $5.9 \mathrm{~s}$. The third order polynomial fits used for profile-analysis are shown as dashed lines. 


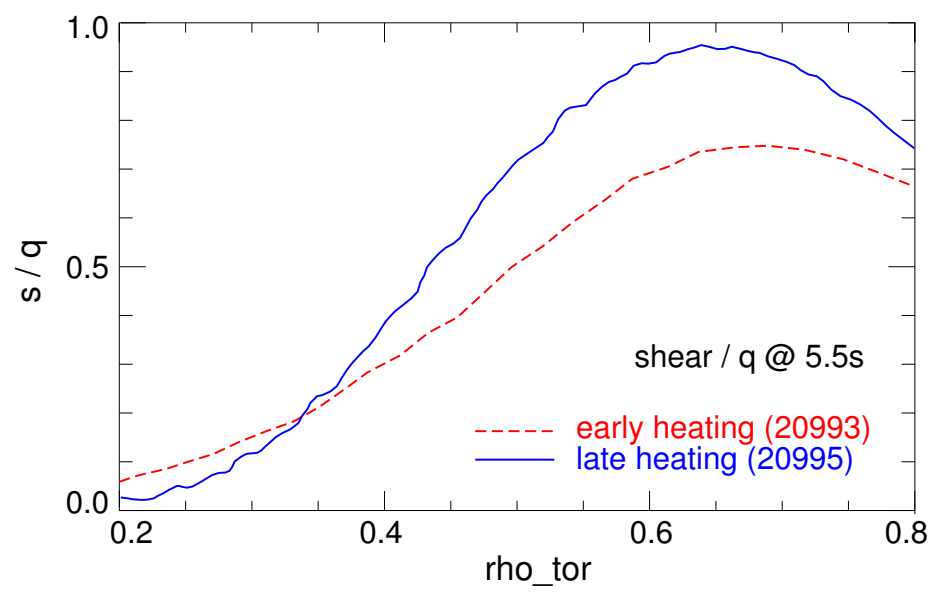

Figure 12. Magnetic shear $s$ divided by $q$ for the early (red, dashed) and late (blue, solid) heated discharges, also shown in figures 2,6,9. From figure 6 it is clear that the difference between the underlying q-profiles is only significant in the range $0.3<\rho_{\text {tor }}<0.6$. Therefore, the differences of the $s / q$-profiles should only be interpreted in this range.

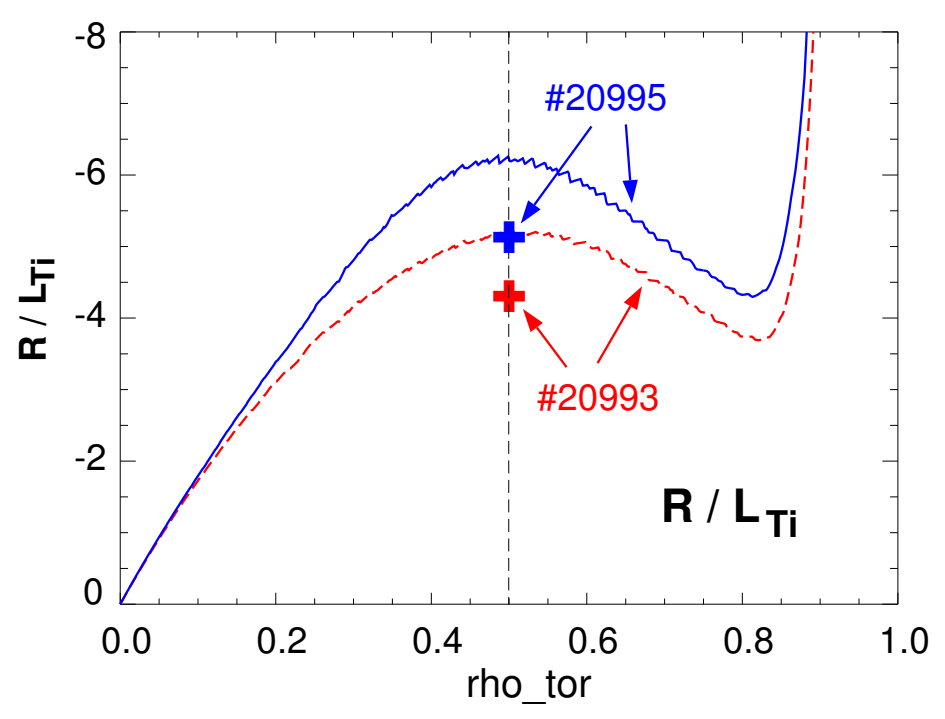

Figure 13. Curves correspond to $R / L_{T i}$ calculated from the fits to the experimental $T_{i}$-profiles as shown in figure 11 for the early-heated discharge \#20993 (red, dashed) and the late heated discharge \#20995 (blue, solid). Crosses correspond to critical values of $R / L_{T i}$ obtained for the onset of ITG turbulence by the linear gyro-kinetic GS2 code at the indicated radial position $\left(\rho_{t o r}=0.5\right)$ for both discharges. 


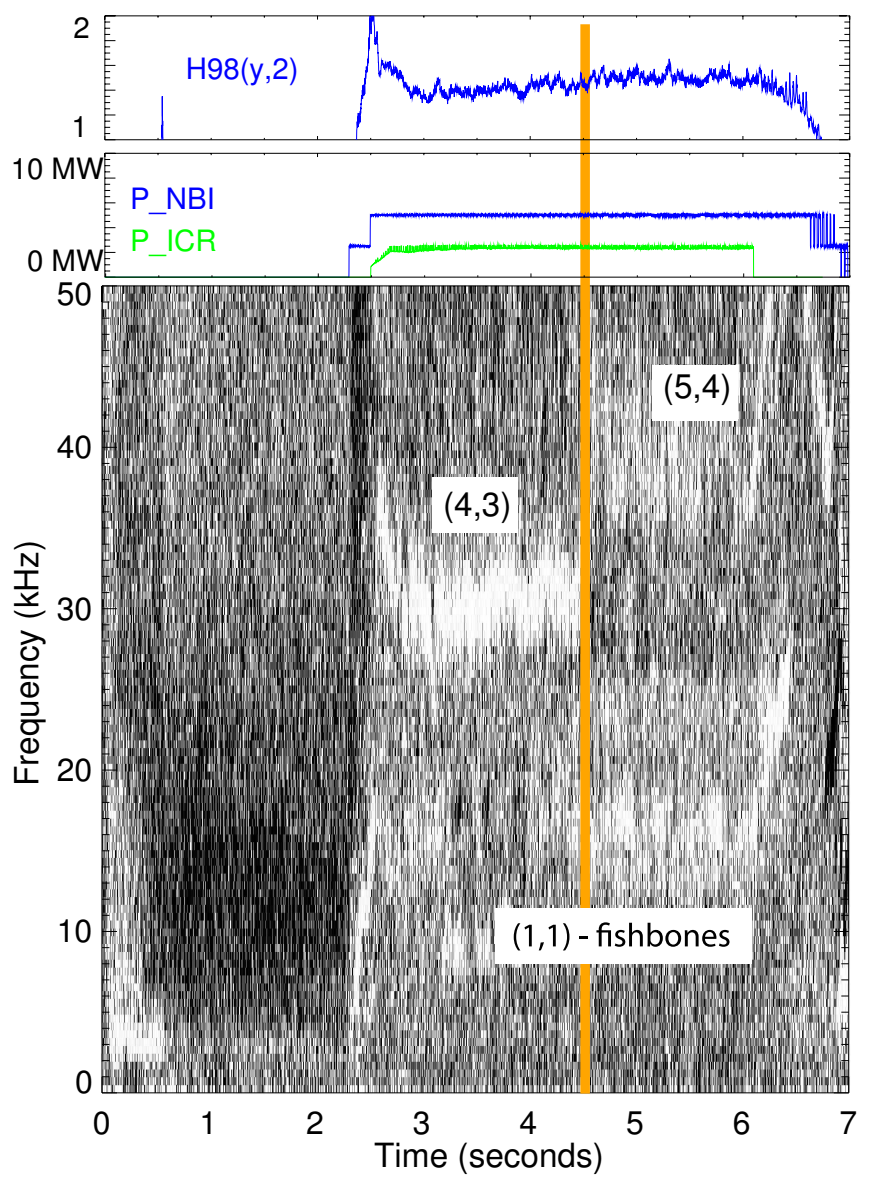

Figure 14. Late heating at $q_{95}=4.0$ ( $\left.\# 20997\right)$ : time traces and MHD activity. From 2.5 to $4.5 \mathrm{~s}$ fishbone activity increases. Finally the NTM changes from $(4,3)$ to $(5,4)$. 